\title{
Mathematical Modeling on the Obesity Dynamics in the Southeastern Region and the Effect of Intervention
}

\author{
Laxmi P. Paudel \\ Department of Mathematics and Computer Science, Albany State University, United States
}

Copyright $\mathrm{C} 2019$ by authors, all rights reserved. Authors agree that this article remains permanently open access under the terms of the Creative Commons Attribution License 4.0 International License

\begin{abstract}
Overweight and obesity has been a major health problem in the United States. The severity is highest in the southeastern region. Social contagion is a significant factor for the progression of the obesity and its identification and control may lead to effective planning in the intervention of the obesity epidemic. In this paper, we devise a SIR model that capture the current dynamics of obesity in the southeastern region of the United States. We discuss the spread of obesity among friends and relatives through social network. With the help of the mathematical model, we discuss the effectiveness of the current intervention programs to control the obesity. We also purpose some affirmative actions to the public health policy makers, the city planning authority, and the community itself that could minimize and even reverse the pattern of obesity.
\end{abstract}

Keywords Overweight, Obesity, Social Contagion, Sedentary, Transmission, Intervention

\section{Introduction}

The prevalence of obesity has increased worldwide in an alarming rate for the last four decades. It is now considered as a global pandemic [1]. 71.6 percent of adults aged 20 and over are overweight or obese. Almost 20 percent of children between the ages of 6 and 19 are obese [2]. It is evident that obesity must be controlled, because it is one of the most important risk factors for many chronic disease [1]. Christakis and Fowler [3] demonstrated that eating pattern and engagement in the physical activities are contagious and obesity can spread from person to person through social network. In the SIR model devised by Ejima in [4], it is shown that social contagion is the significant factor for the increase in the obesity, and obesity spread has shown the pattern of infectious disease.

In this paper, we briefly introduce obesity, its causes and the effects. We use the SIR model to capture the current dynamics of obesity in the southeastern region of the United States and show mathematically that intervention program that can check contagious factor of obesity would effectively contribute to control or lower the prevalence of the obesity.

Obesity is defined as condition of abnormal or excessive fat accumulation in adipose tissue, to the extent that the health is impaired [1]. Accumulated excess fat around waist and abdominal region, or peripherally distributed around the body may indicate a lot about overall health condition [5].

The body mass index (BMI) is widely used and commonly accepted parameter to classify the degree of adiposity or body fatness. It is defined as the ratio of weight of an individual in kilograms and the square of the height in meters $\left(\mathrm{kgm}^{-2}\right)$. As per the current World Health Organization (WHO) criteria, the numerical value of BMI in adults is interpreted as follows: BMI less than $18.5 \mathrm{kgm}^{-2}$ is considered underweight, from $18.5 \mathrm{kgm}^{-2}$ to $24.9 \mathrm{kgm}^{-2}$, ideal weight, $25 \mathrm{kgm}^{-2}$ to $25.9 \mathrm{kgm}^{-2}$, overweight, and 30 $\mathrm{kgm}^{-2}$ and above is obese. The obese category is subdivided into obese class I, which is BMI from $30 \mathrm{kgm}^{-2}$ to 34.9 $\mathrm{kgm}^{-2}$, obese class II, $35 \mathrm{kgm}^{-2}$ to $39.9 \mathrm{kgm}^{-2}$, and obese class III higher than $40 \mathrm{kgm}^{-2}$.

To determine the overweight and obesity in children and teens relative measure of BMI is used [2]. Overweight is defined as a BMI at or above $85^{\text {th }}$ percentile and below the $95^{\text {th }}$ percentile for the children and teens of the same age and sex whereas, obesity as the BMI at or above the $95^{\text {th }}$ percentile. The children's body composition varies as they grow and the variation is different between boys and girls. So relative BMI level is used with respect to the same age and sex. In the United States, CDC Growth Charts are the most commonly used tools to determine the size and growth patterns of children and teens.

Obesity has a wide range of negative effects on health. Every year obesity related conditions cost over 150 billion dollars in the health care and causes an estimated 300,000 premature deaths in the Unites States alone [6].

Adult obesity is associated to three to four time higher risk of morbidity due to cardiovascular disease than in the 
general population [7]. Atherosclerosis (hardening of the arteries) is present 10 times more often in obese people compared to those who have normal body fat [8]. High body weight is associated to elevated cholesterol level.

Elevated cholesterol level leads to fatty deposit on blood vessels and causes chest pain and heart attack. Narrowed arteries can lead to blood clot and Stroke. Type 2 diabetes, metabolic syndrome, gall bladder disease and certain cancers like breast, prostate and colon cancers are highly associated with obesity [9]. Obesity also carries serious implications for psychological health, mainly due to societal prejudice against fatness [5]. All of these adverse health conditions contribute to huge health care cost and leads to a large number of premature deaths.

The primary cause of obesity is consumption of more calories and burning less through physical activities [10]. Obesity is generally as a result of a complex combination of genetics, metabolic, socioeconomic factors, and lifestyle choices. Some diseases, high stress, endocrine disorders, and medications can also contribute to the obesity [11].

\subsection{Obesity Statistics in Four States of Southeastern Region}

The proportion of the overweight and obese population is alarmingly high throughout the United States, and it is constantly growing for the last four decades. As measured by the National Health and Nutrition Examination Survey (NHANES), most recent national adult obesity rate is approaching 40 percent [9]. Seven states have prevalence of adult obesity above $35 \%$ [2]; West Virginia (38.1\%), Mississippi (37.3\%), Oklahoma (36.5\%), Iowa (36.4\%), Alabama (36.3\%), Louisiana (36.2\%), and Arkansas (35\%). Mississippi, Alabama, Louisiana and Arkansas lie in the southeastern region. They are ranked as second, fifth, sixth, and seventh state by the severity of the adult obesity. The table below provides the adult obesity rates in the region by states from the year 1990.

Table 1. Data Showing the Steady Growth of Obesity in Four Southeastern States since the Year 1990

\begin{tabular}{|c|c|c|c|c|}
\hline Year & Mississippi & Alabama & Louisiana & Arkansas \\
\hline 1990 & $15.0 \%$ & $11.2 \%$ & $12.3 \%$ & \\
\hline 1994 & $19.4 \%$ & $15.7 \%$ & $17.0 \%$ & $16.0 \%$ \\
\hline 2000 & $23.7 \%$ & $22.6 \%$ & $22.6 \%$ & $21.9 \%$ \\
\hline 2003 & $28.1 \%$ & $28.4 \%$ & $24.8 \%$ & $25.2 \%$ \\
\hline 2004 & $28.1 \%$ & $27.7 \%$ & $25.8 \%$ & $25.0 \%$ \\
\hline 2005 & $29.5 \%$ & $28.7 \%$ & $27.4 \%$ & $26.4 \%$ \\
\hline 2006 & $30.6 \%$ & $29.4 \%$ & $28.2 \%$ & $27.0 \%$ \\
\hline 2007 & $31.7 \%$ & $30.1 \%$ & $29.5 \%$ & $28.1 \%$ \\
\hline 2008 & $32.5 \%$ & $31.2 \%$ & $28.9 \%$ & $28.6 \%$ \\
\hline 2009 & $33.8 \%$ & $31.6 \%$ & $31.2 \%$ & $30.1 \%$ \\
\hline 2010 & $34.4 \%$ & $32.3 \%$ & $31.6 \%$ & $30.6 \%$ \\
\hline 2011 & $34.9 \%$ & $32.0 \%$ & $33.4 \%$ & $30.9 \%$ \\
\hline 2012 & $34.6 \%$ & $33.0 \%$ & $34.7 \%$ & $34.5 \%$ \\
\hline 2013 & $35.1 \%$ & $32.4 \%$ & $33.1 \%$ & $34.6 \%$ \\
\hline 2014 & $35.5 \%$ & $33.5 \%$ & $34.9 \%$ & $35.9 \%$ \\
\hline 2015 & $35.6 \%$ & $35.6 \%$ & $36.2 \%$ & $34.5 \%$ \\
\hline 2016 & $37.3 \%$ & $35.7 \%$ & $35.5 \%$ & $35.7 \%$ \\
\hline 2017 & $37.3 \%$ & $36.3 \%$ & $36.2 \%$ & $35.0 \%$ \\
\hline Rank in 2017 & 2 & 5 & 6 & 7 \\
\hline
\end{tabular}




\subsection{Graph of the Obesity Trend}

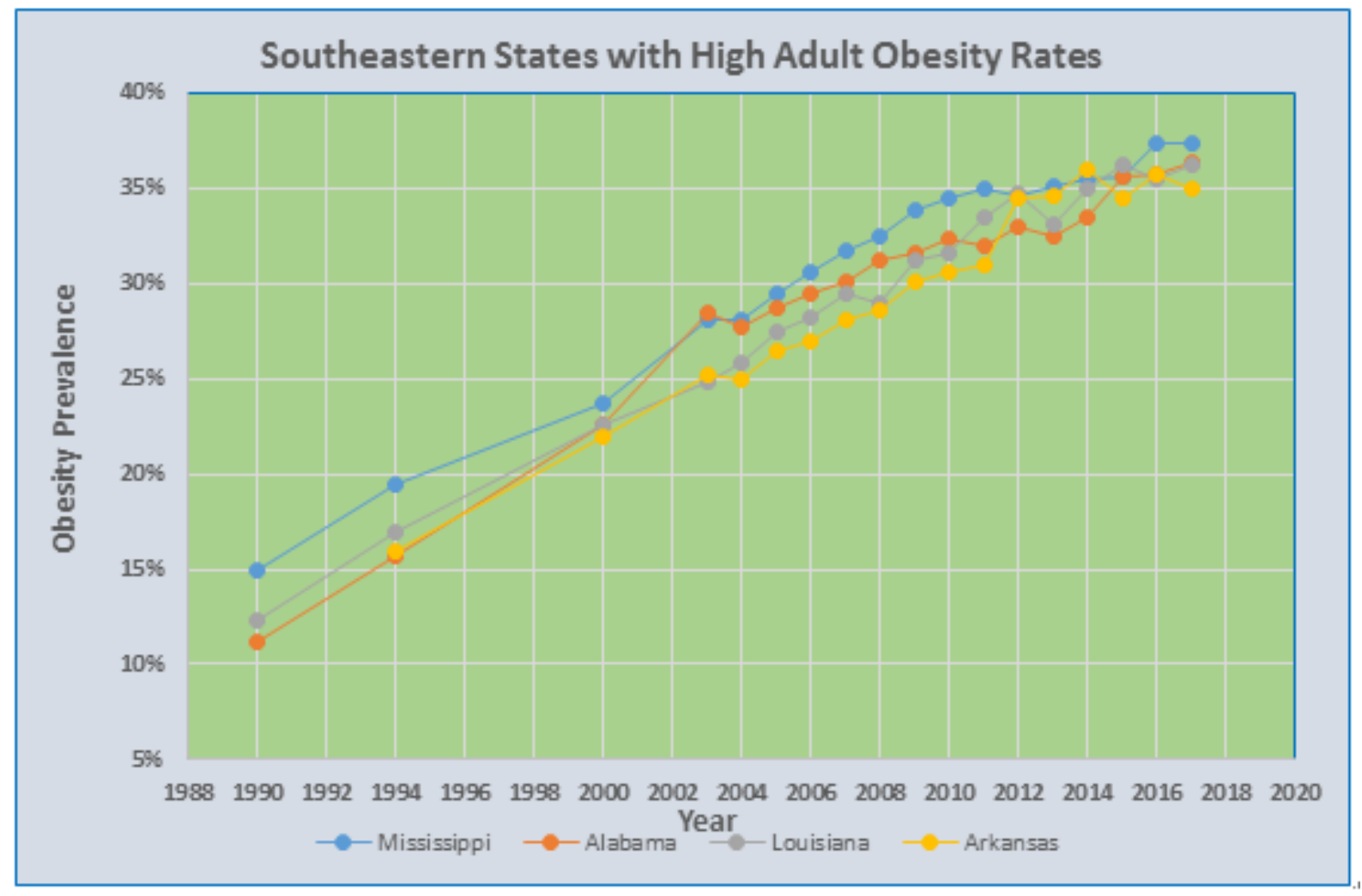

Figure 1. The adult obesity constantly inreasing.

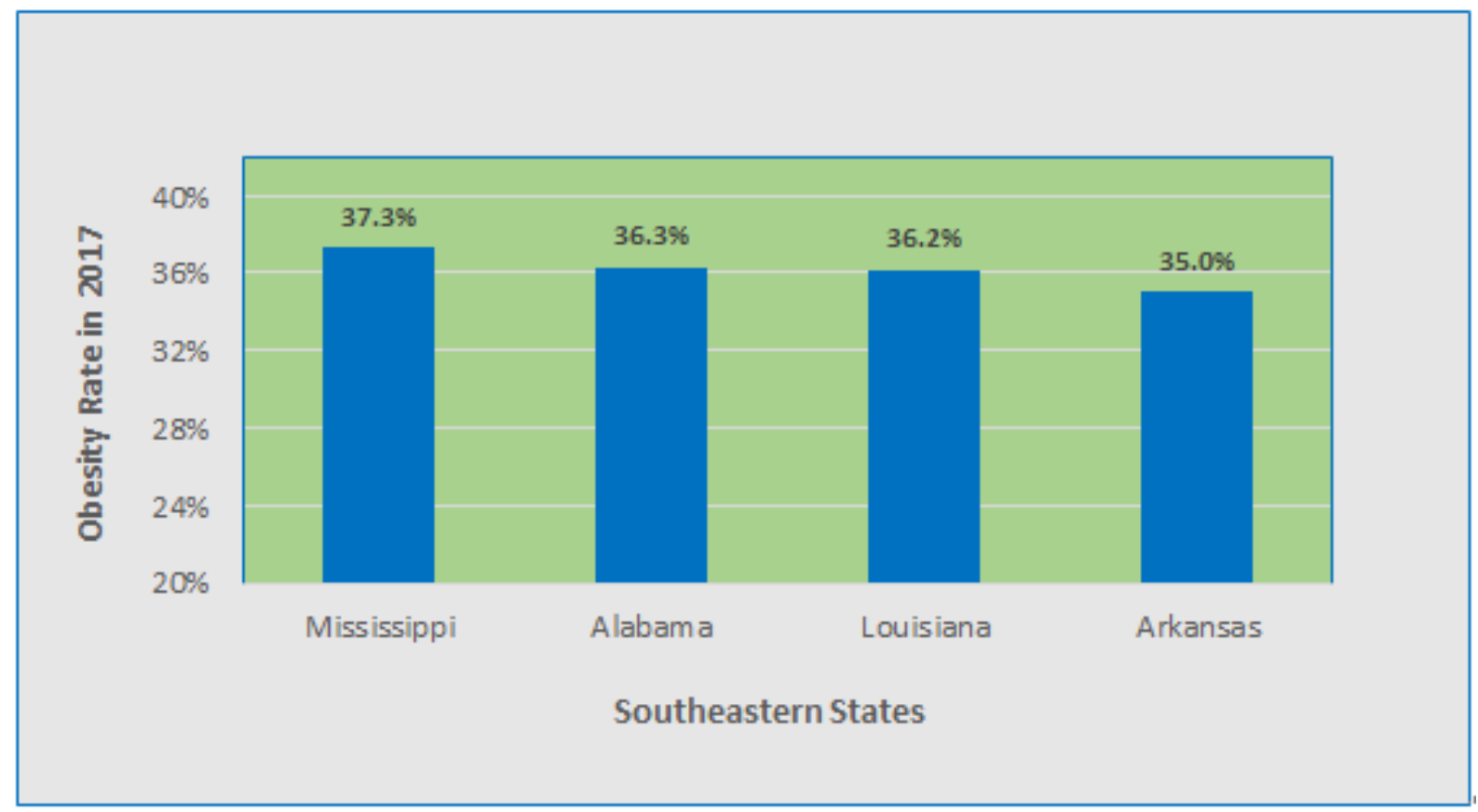

Figure 2. Current Obesity Rates, all at or above $35 \%$. 


\subsection{Effect of Social Contagion on Obesity}

There is growing evidence in literature that the growth pattern of obesity is like that of a contagious disease [12]. When friends and family members are obese, or a family moves to an obese community, an individual would be susceptible to weight gain through social contagion [10]. An epidemiological model can be used to represent the growth pattern. We use SIR model to describe the pattern of obesity, and our study primarily focuses in the Southeastern region of the United States.

\subsection{SIR Model for the Obesity Growth}

In this model never obese individuals have tendency to be obese and they are taken as susceptible. Obesity is assumed to be contagious and obese people are taken as infectious, and $\mathrm{x}$-obese individuals as recovered. We represent them as functions of time, namely, $\mathrm{S}(\mathrm{t}), \mathrm{I}(\mathrm{t})$ and $\mathrm{R}(\mathrm{t})$ respectively. Some other symbols used in the model are as follows.

$\mathrm{N}$ : population size, assumed to be constant

$\mathrm{N}(\mathrm{t})=\mathrm{S}(\mathrm{t})+\mathrm{I}(\mathrm{t})+\mathrm{R}(\mathrm{t})$ $\mu$ : The birth and death rate

$\beta$ : The transmission coefficient of obesity

$\epsilon$ : The hazard of obesity due to non-contagious reasons

$\gamma$ : The natural recovery rate

$\sigma$ : Relative risk of regaining the weight among ex-obese individuals

$\lambda(t)=\beta I(t)+\epsilon:$ Hazard rate of obesity or force of infection

The value of $\sigma$ is greater than 1 , as ex-obese individuals have stronger tendency to fall back to obesity as compared to the individuals who were never obese. The empirical value of the relative risk from literature is approximately 8.0 [4]. The force of infection $\lambda$ has two components; $\beta I(t)$, the hazard of obesity through contagious route and $\epsilon$, the hazard of obesity for non-contagious reasons. Eating habit and physical activity level acquired with constant touch and impression with family and friends affects the value of $\beta$. Many factors like genetics, dietary habit and other lifestyle components are included in $\epsilon$. For simplicity, as a whole $\epsilon$ is assumed to be constant.

\subsection{Diagram to Represent the Pattern of Obesity}

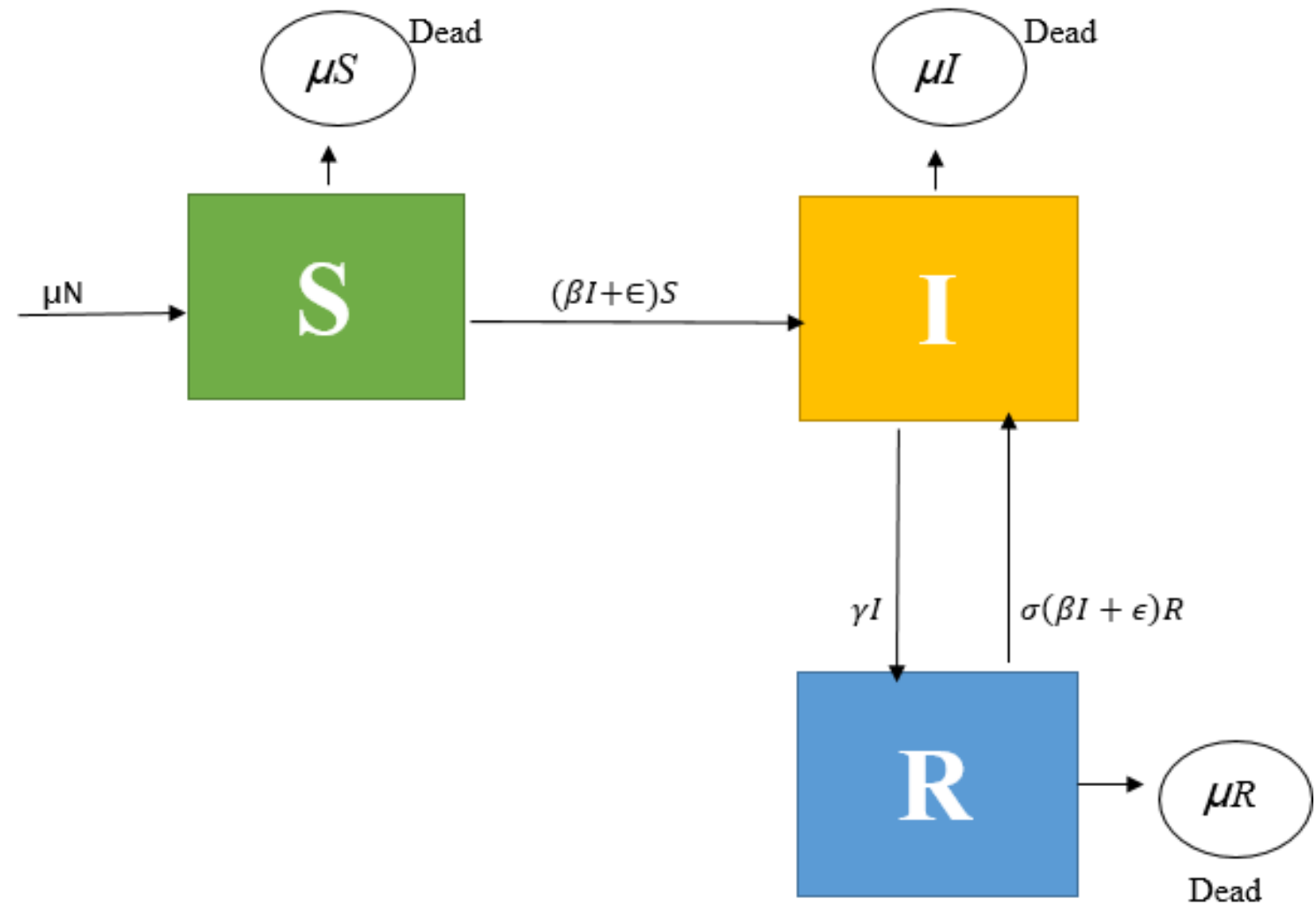




\section{Methods}

The epidemiological process of becoming obese and recovering from obesity are represented as the functions of time. We consider both contagious and non-contagious factors that contribute to the obesity. The dynamics of obesity given in the above diagram can be represented by the following system of ordinary differential equations (ODE) [4].

$$
\begin{gathered}
\frac{d S}{d t}=\mu N-[\beta I(t)+\varepsilon] S(t)-\mu S(t) \\
\frac{d I}{d t}=[\beta I(t)+\varepsilon] S(t)+\sigma[\beta I(t)+\varepsilon] R(t)-(\mu+\gamma) I(t) \\
\frac{d R}{d t}=\gamma I(t)-\sigma[\beta I(t)+\varepsilon] R(t)-\mu R(t)
\end{gathered}
$$

The above system of equations can be numerically solved with initial condition $\mathrm{S}(0)=\mathrm{N}$.

Solving $d(S, I, R) / d t=0$, we get asymptotically equilibrium solution point $\left(S^{*}, I^{*}, R^{*}\right)$, where all the trajectories of the system converge.

Table 2. Values of the parameters

\begin{tabular}{|c|c|c|c|}
\hline Description & Notation & Baseline Value & Reference \\
\hline Population size & $\mathrm{N}$ & 100,000 & Assumed, as in [4] \\
\hline Average life expectancy at birth & $1 / \mu$ & 75.5 Years & {$[4]$} \\
\hline Transmission Coefficient of obesity & $\beta$ & $1.99 \times 10^{-7}-4.33 \times 10^{-7}$ & {$[4],[7]$} \\
\hline Non-contagious hazard of obesity & $\varepsilon$ & $\begin{array}{c}0.012 \\
\text { (per year) }\end{array}$ \\
\hline Relative hazard of obesity among ex-obese & $\sigma$ & $5.0-8.0$ & {$[4]$} \\
\hline Average duration of obesity & $1 / \gamma$ & 35.8 (Years) & {$[4],[13]$} \\
\hline
\end{tabular}

The values of the parameters were estimated in such a way that the trajectories show a good fit through prevalence data from 1990-2000. We take the value of $\mathrm{N}$ to be constant and equal to 100,000 to keep the model simple. The average life expectancy at birth and the value of the other parameters are consistent with empirical values in the literature.

\subsection{Pattern of Obesity by SIR Model}

The numerical solution of the system of differential equations in the SIR model are obtained as shown in the picture below.

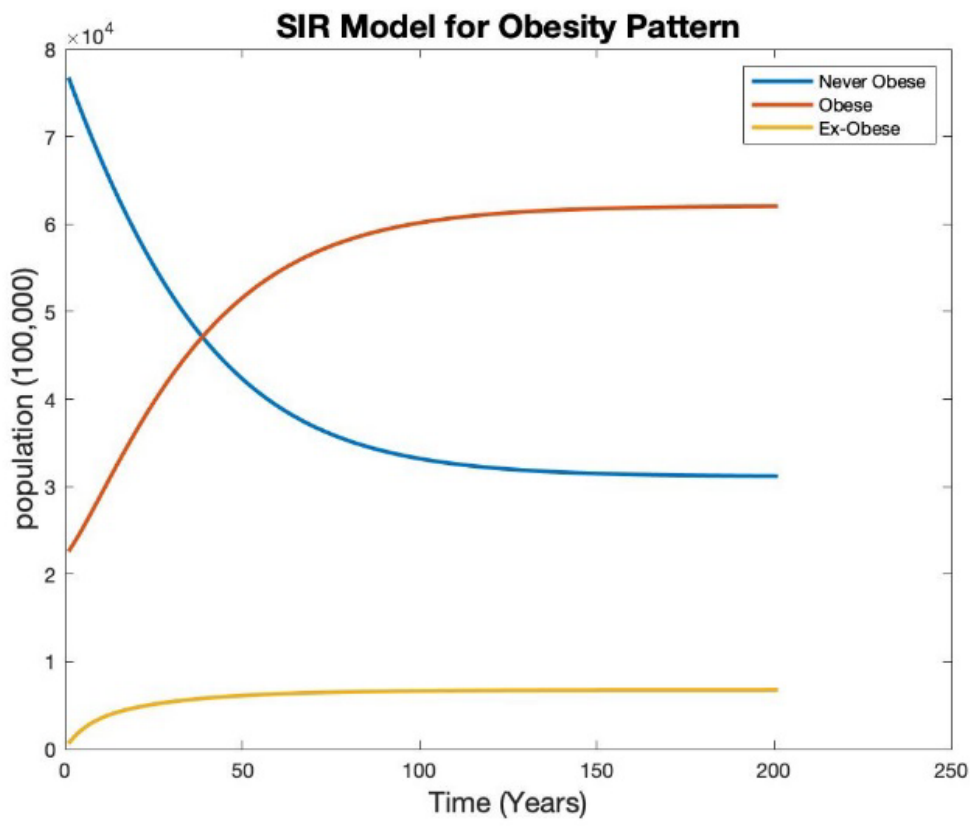

Figure 3. SIR Model Obesity Pattern 
The figure shows the baseline dynamics of an obesity epidemic. These are the time dependent epidemiological trajectories. The blue graph represents the never-obese population, red graph the obese population, and the orange graph the ex-obese ones. The number of the never obese individuals are high because it has been assumed that all the new born are non-obese. As per the graph, as time passes by the prevalence of obesity converges to an equilibrium level. It takes almost 200 years to reach the equilibrium state of obesity.

\subsection{Level 1 Intervention}

Level 1 intervention is a hypothetical intervention program that affects both the contagious and non-contagious hazard of obesity and lowers the overall hazard rate of obesity $\lambda(t)=\beta I(t)+\epsilon$. The intervention program includes early screening for overweight and obesity, healthy lifestyle changes aiming to maintain a healthy weight. It also includes taking part in the weight loss program and maintaining the healthy weight after the intentional weight loss is achieved.

We assume that the level 1 intervention keeps the value of transmission coefficient of obesity $\beta$ at the lower end which is $1.99 \times 10^{-7}$ from various literatures. It reduces the relative hazard of obesity $(\sigma)$ among ex-obese individuals from 8.0 to 5.0, which makes sense because with constant counselling and consistent engagement of active lifestyle and healthy eating, weight loss can be permanent.

\subsection{Comparison of Obesity Growths with and without the Intervention}

The adjacent set of graphs below show the prediction of prevalence of obesity by the current trend and the prediction of obesity after the level 1 intervention program. In all states, the intervention program significantly reduces the obesity rates.

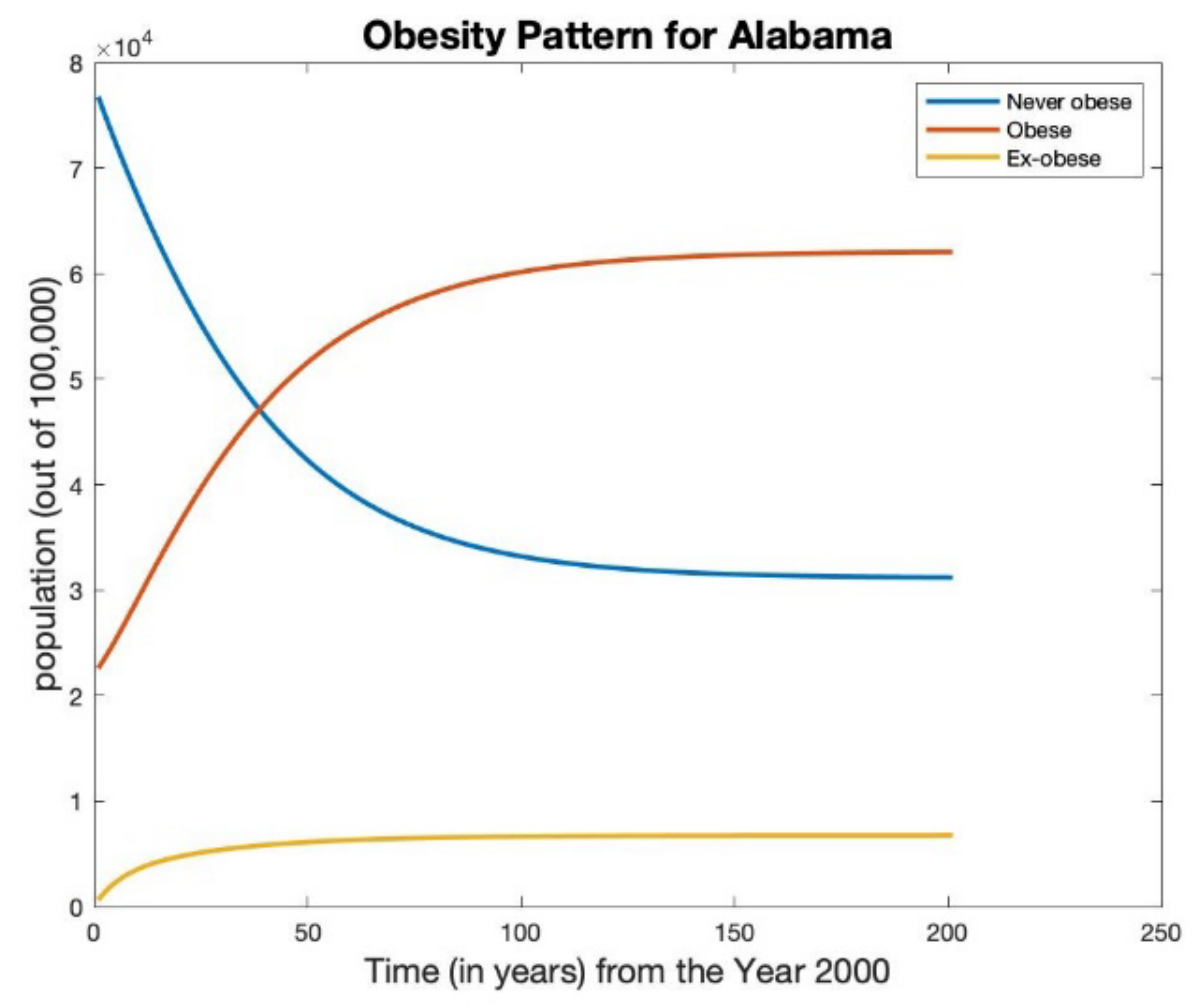

Figure 4. Obesity Pattern for Alabama 


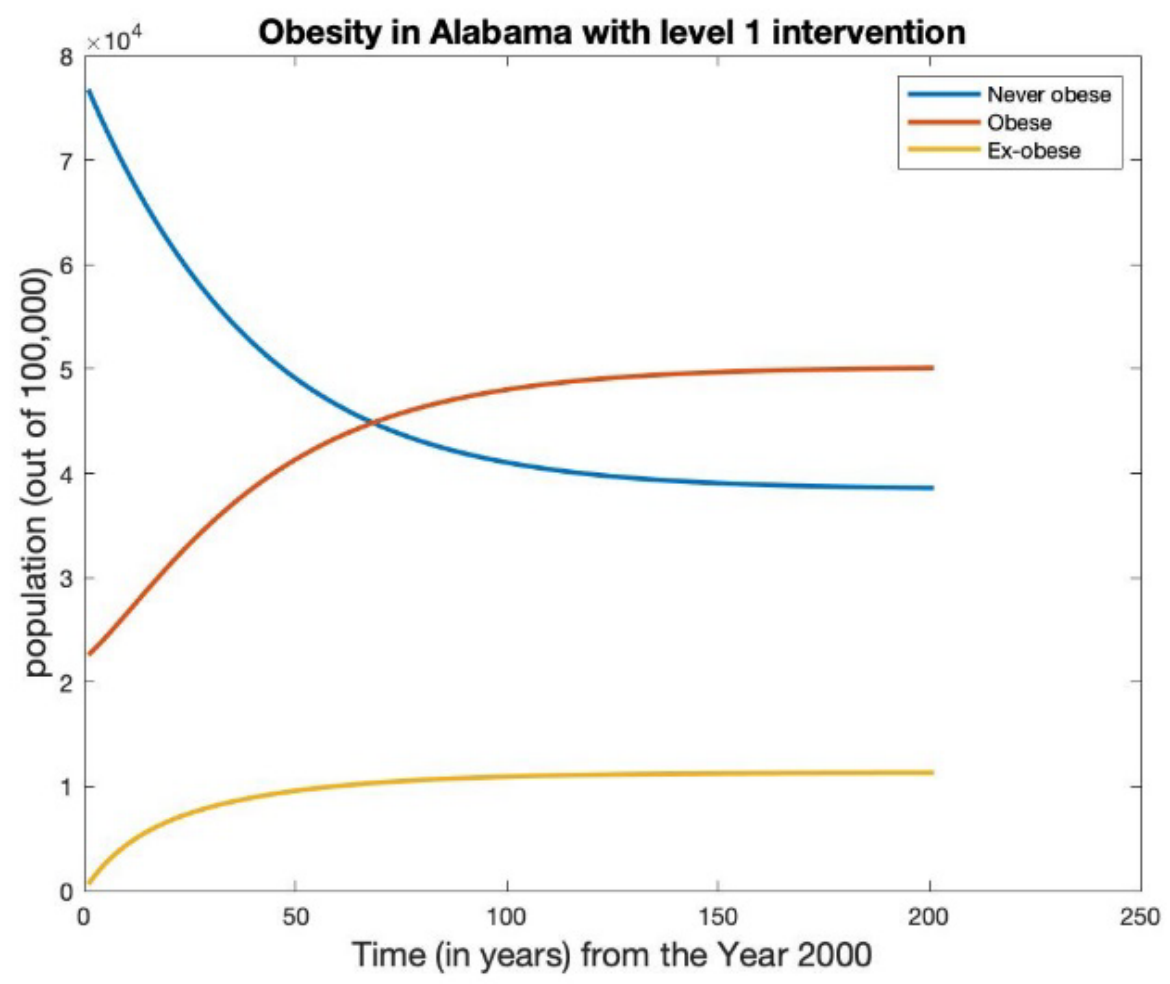

Figure 5. Obesity in Alabama with Level 1 Intervention

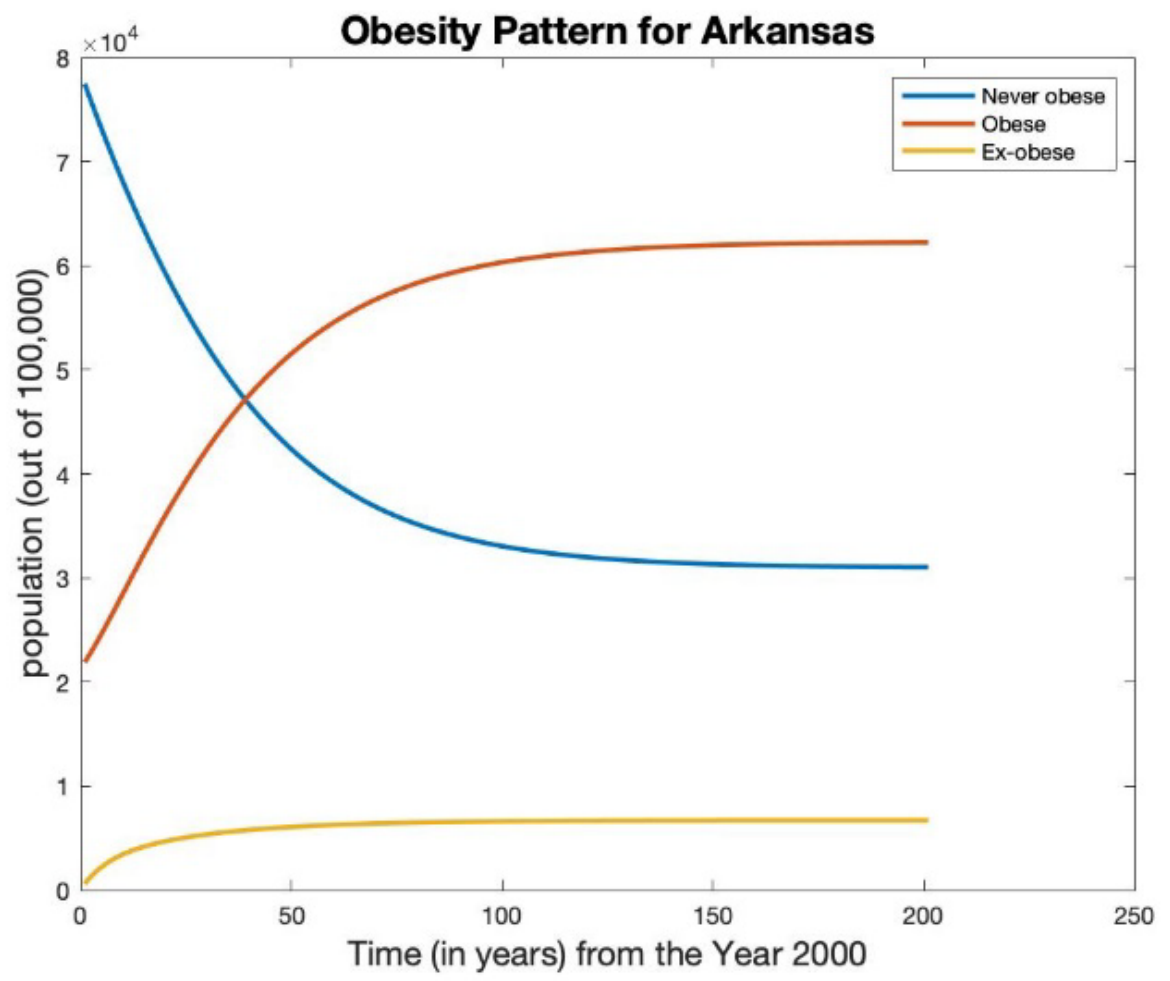

Figure 6. Obesity Pattern for Arkansas 


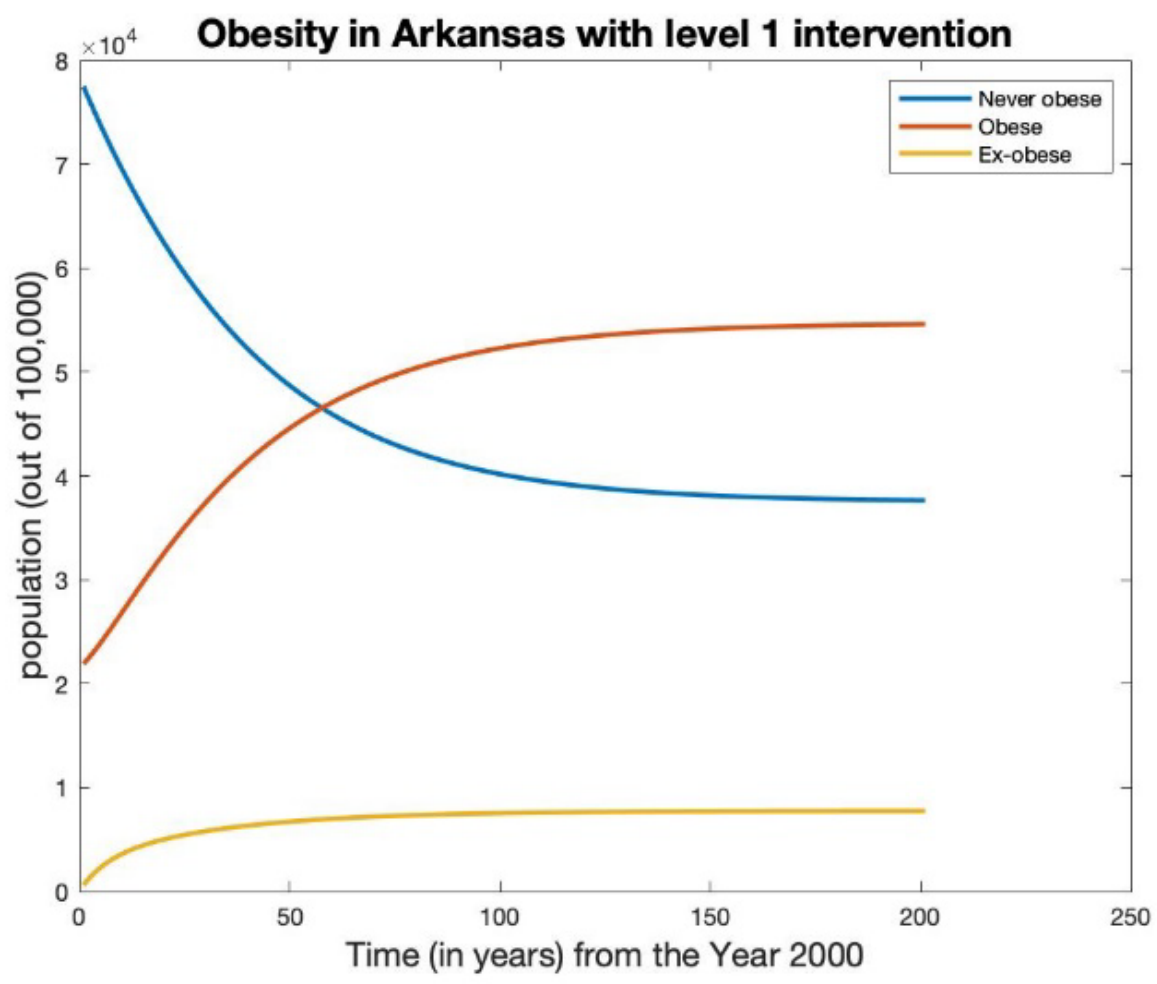

Figure 7. Obesity in Arkansas with level 1 Intervention

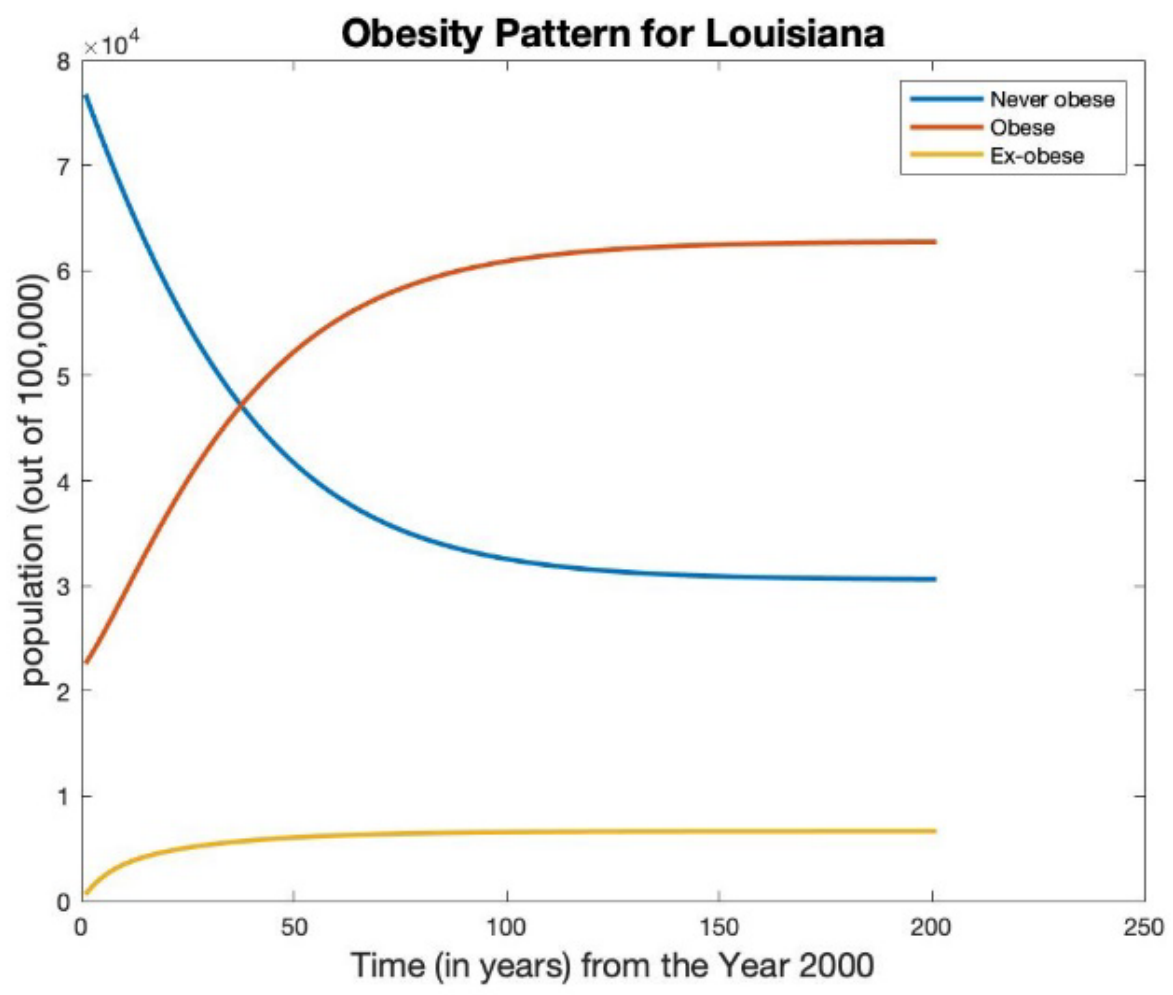

Figure 8. Obesity Pattern for Louisiana 


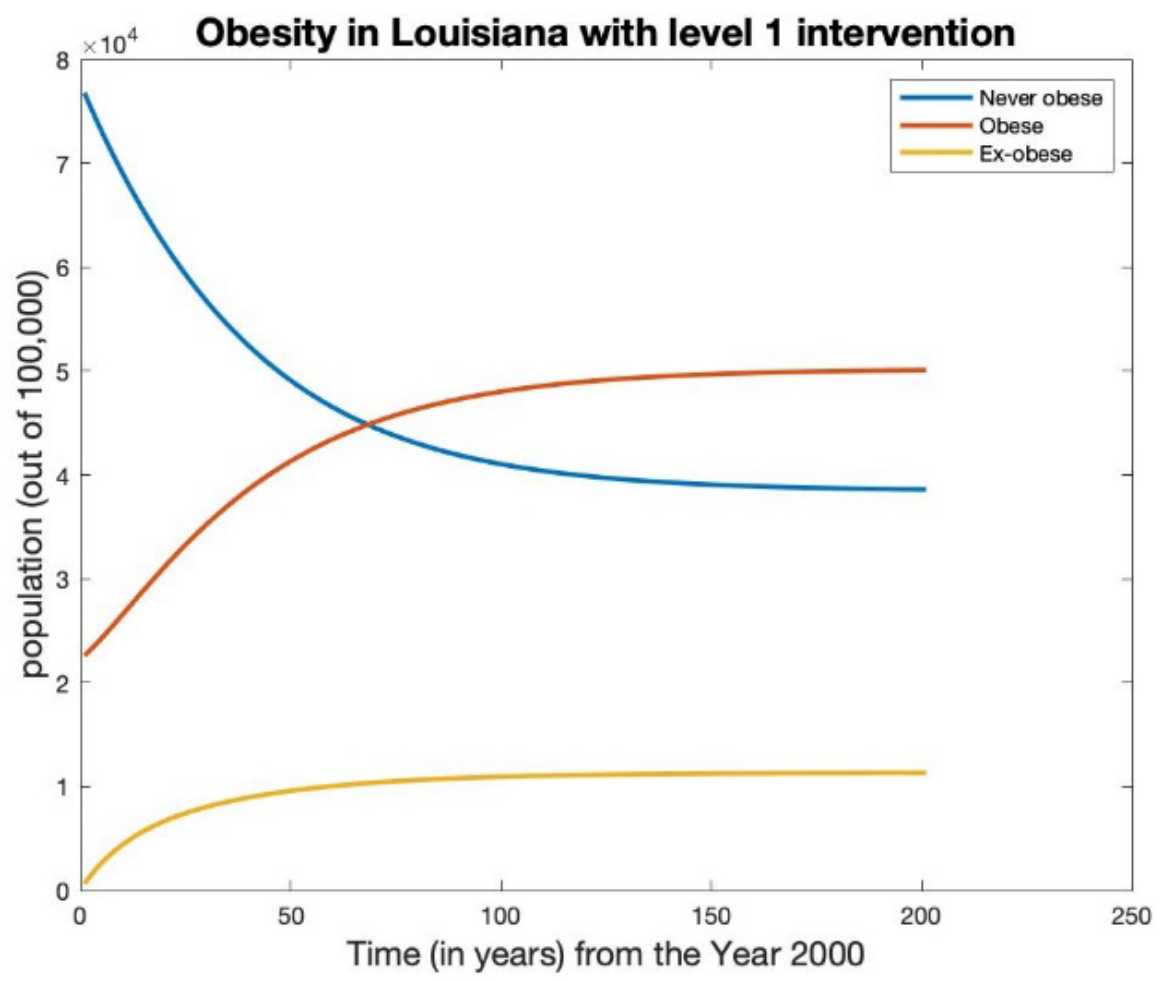

Figure 9. Obesity in Louisiana with level 1 intervention

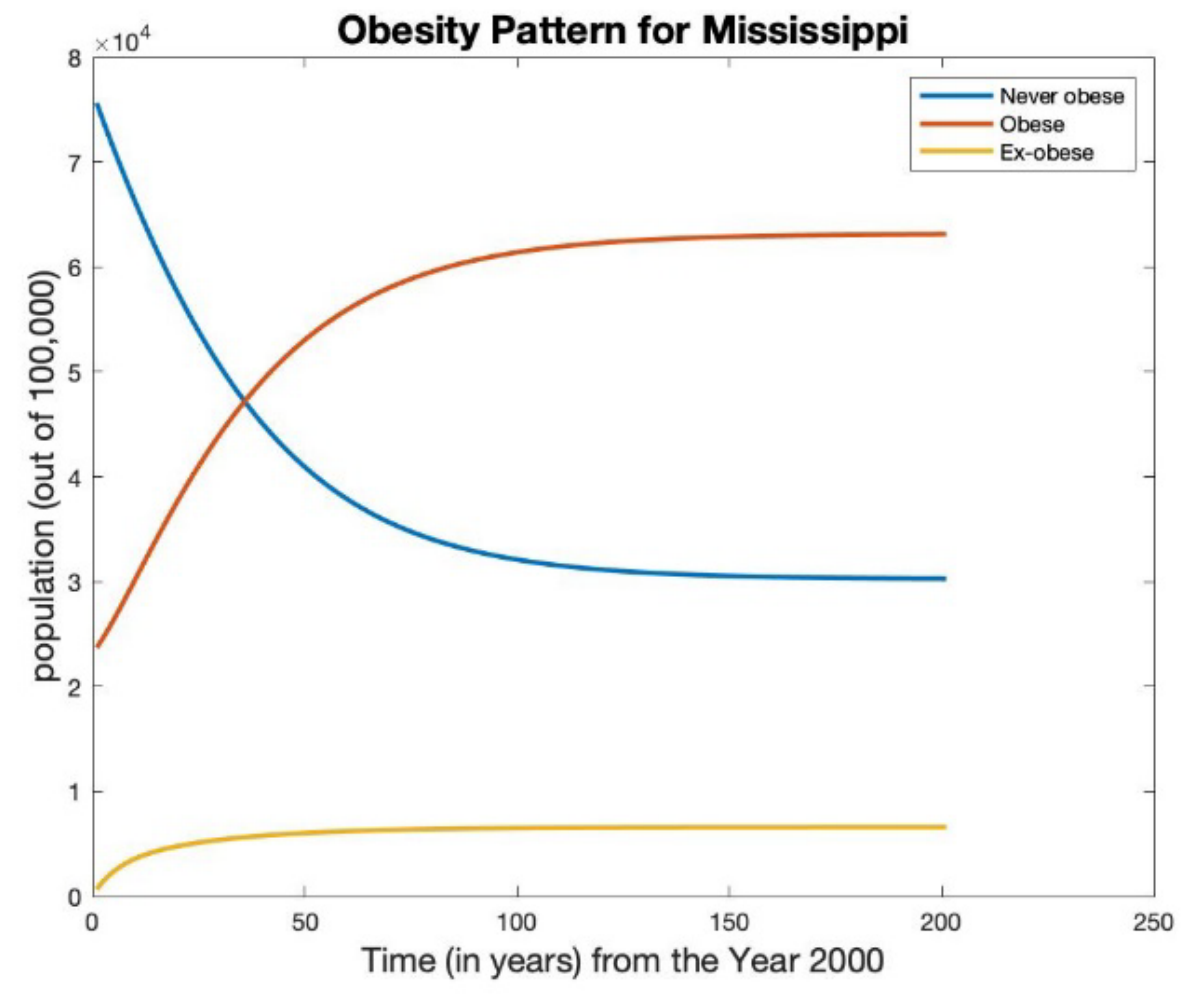

Figure 10. Obesity Pattern for Mississippi 


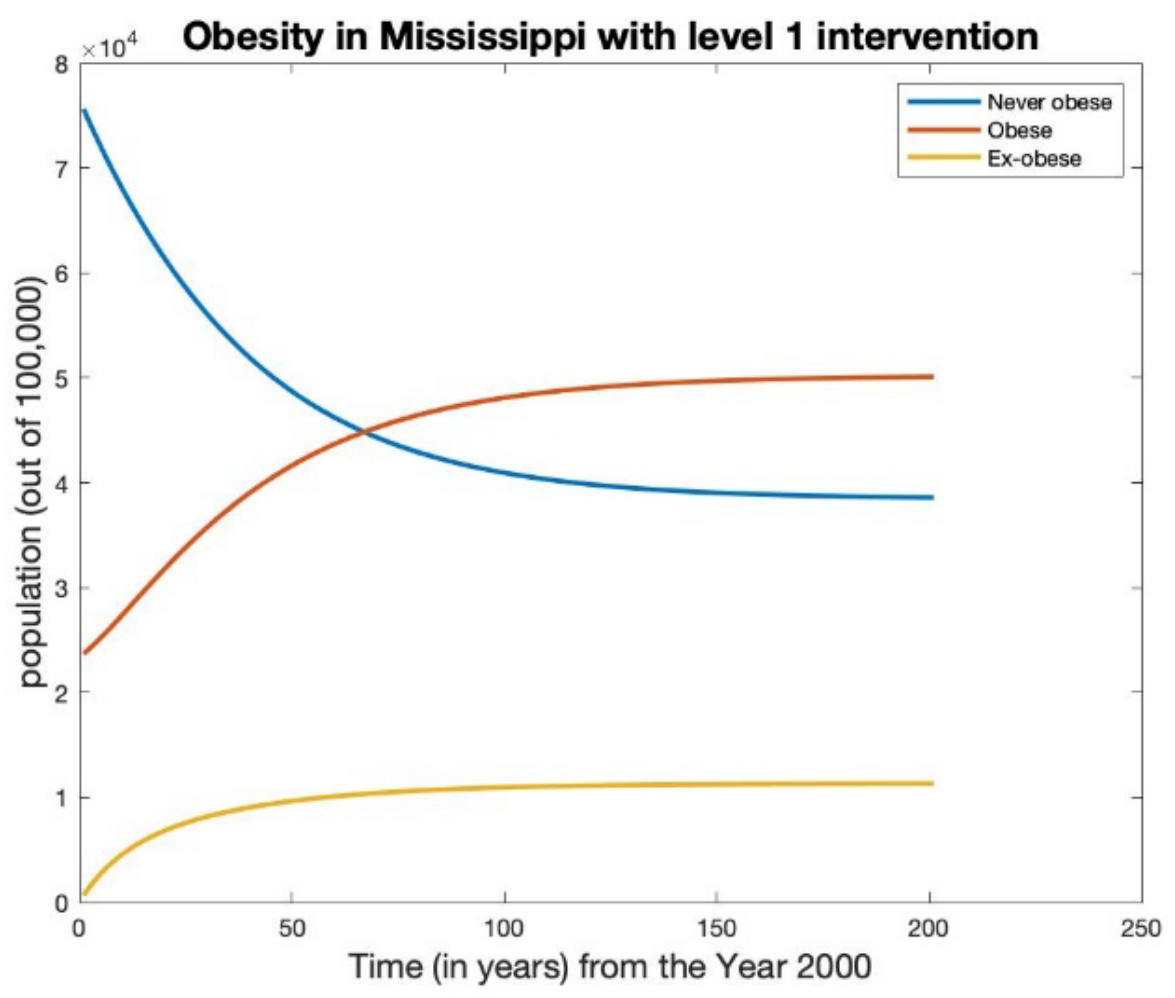

Figure 11. Obesity in Mississippi with Level 1 Intervention

Table 3. Obesity Rates Predicted by the Model in the Year 2100 with or without Intervention

\begin{tabular}{|c|c|c|c|}
\hline State & Year 2000 (base year) & Year 2100 (Expected by the current trend) & Year 2100 (Expected after level 1 Intervention) \\
\hline Mississippi & $23.70 \%$ & $62 \%$ & $50.29 \%$ \\
\hline Alabama & $22.60 \%$ & $62.04 \%$ & 50.09 \\
\hline Louisiana & $22.60 \%$ & 62.05 & 50.1 \\
\hline Arkansas & $21.90 \%$ & 62.2 & 50.28 \\
\hline
\end{tabular}

Results: Note that the percentage of obese adults can be lowered in Mississippi, Alabama, Louisiana, and Arkansas by $11.7 \%, \quad 11.9 \%, \quad 12.04$, and $11.92 \%$ respectively by the year 2100 with the help of level 1 intervention.

\subsection{Treating Obesity}

It is very crucial to have a BMI in healthy range [14]. Obesity is a complex problem and there is no single panacea that works for all [15]. We need a multifaceted approach to deal with it. Healthy food must be accessible to all common people and we all need to practice a healthy eating style [16]. There are many ways to eat healthfully and everyone has their own eating style. We should make healthier choices that reflect our preference, culture, traditions, and budget. Fruits, vegetables, grains, dairy, and protein must be included to get the most nutrition and meet the personal calorie needs [17]. We should aim a variety of foods and beverages from each food group and limit saturated fat, sodium, and added sugars. For already obese people, individualized weight loss plan must be created and implemented.

\subsection{Weight Loss Strategies and Their Effectiveness}

Weight loss requires a lot of patience and maintaining a healthy weight requires a lifelong commitment. Losing weight slowly will be more effective and healthy over the long term because quick weight loss often spurs weight regain. Weight loss strategies may include dietary therapy, physical activity, and lifestyle change.

\subsection{Strategies for Obesity Prevention}

Prevention is the key to controlling the obesity epidemic. Research has shown that overweight children have higher chance of being obese adults when they grow. Parents, health care professionals, schools, city planning authority, state, and federal government has to put collaborative 
effort to prevent and control obesity.

World Health Organization (WHO) has put forward various obesity prevention strategies. Public health approaches are directed at all members of the community [1]. There are selective approaches that covers at high risk individuals and groups. A targeted approach is directed at the individuals that already have weight related problems and diseases. The population-wide obesity prevention programs have greater potential to counter obesity and are more cost effective than the clinic based treatments [5].

Centers for Disease Control and Prevention (CDC) have a Division of Nutrition, Physical Activity and Obesity (DNPAO) that has taken a pioneer role of controlling the obesity in the United States. DNPAO supports healthy eating, active living. Its initiatives include the fighting obesity by creating healthy child care centers, hospitals, schools, and worksites. It conducts and supports research, surveillance and evaluation studies to keep Americans healthy and strong at every state of life.

The following strategies are recommended for the prevention and the control of obesity.

Effort from Parental Level: Parents can change the family eating habit, and increase physical activity levels. Parents are role model for children. If parents eat healthy food with children in the family dining table, children are more likely to do the same.

Community Efforts: Community can support healthy eating and active living in a variety of settings as follows.

Early Care and Education: Environment in the childcare and early education facilities may directly impact what children consume and how active they are. Young children must be encouraged to drink water rather than sugar sweetened beverages. They must be served with fruits, vegetables, whole grains, and nonfat or low-fat dairy products for their food. The kids should be given enough play time to stay active during their time in the facilities.

School Community: In addition to serving healthy food, and free water, schools can limit the students' access to sugary beverages and processed snacks. The amount of time that students are being moderately to vigorously physically active during physical education classes may be increased. All the students should be encouraged and given opportunity to take part in the games such as soccer, basketball, football that require vigorous physical activity. Schools should invest to build walking and running tracks around school facility.

Worksites: Similar to the school community, worksites should provide its employee active living environment and healthy eating options. Healthy food service guidelines must be enforced to make the healthier food and beverages more accessible than poor dietary options. Walking sites and gym after work can be good options. Employee can be given incentives for periodically participating active living practices.

Healthy Food Environments: The state and federal government can allocate a budget to provide incentives for supermarkets and famers markets to establish their business in the underserved areas. They can make policies for nutrition standards for childcare, schools, hospitals and workplaces and provide incentives for its implementation.

Active Living Environment: City planning authority should build walking tracks, parks, and recreation centers around the residential areas. Side tracks to get to schools, grocery stores, gym and other recreational facilities can significantly promote active living.

\section{Conclusions}

Obesity is continuously rising in the last four decades in an epidemic level. It is more severe in the southeastern region of the United States. Easy access to fast foods, sedentary life style, and extensive use of technology has contributed to the rapid growth of obesity. Obesity can be controlled and even reversed, but it requires a strong commitment and a multifaceted approach. The presented SIR model can help to estimate the prevalence of obesity dynamics. It can also be used to estimate the effectiveness of the obesity control programs. As per the model, identification of the socially contagious nature of obesity can help to devise effective intervention program to manage and counter obesity.

\section{REFERENCES}

[1] World Health Organization. Obesity: preventing and managing the global epidemic. Report of a WHO consultation. World Health Organ Tech Rep Ser. 2000; 894: $1-253$.

[2] Overweight and Obesity, Centers for Disease Control and Prevention website. https://www.cdc.gov/obesity/data/adul t.html Accessed February 16, 2019.

[3] What is the Disease of Obesity? AACE Obesity Resource Center website.http://obesity.aace.com/what-is-the-disease -of-obesity . Accessed March 4, 2019.

[4] Ejima et al: Modeling the obesity epidemic: social contagion and its implications for control. Theoretical Biology and Medical Modelling 2013 10:17.

[5] F. Ofei, Obesity - A preventable disease, Ghana Med J September 2005; Volume 39 (3):98-101.

[6] Hales CM, Carroll MD, Fryar CD, Ogden CL. Prevalence of obesity among adults and youth: United States, 20152016. NCHS data brief, no 288. Hyattsville, MD: National Center for Health Statistics. 2017.

[7] Hill AL, Rand DG, Nowak MA, Christakis NA: Infectious Disease Modeling of Social Contagion in Networks. PLoS Comput Biol. 2010, 6: e1000968-10.1371/journal.pcbi.100 0968.

[8] Obesity Prevention. Stanford Health Care website. https://s tanfordhealthcare.org/medical-conditions/healthy-living/ob 
esity/prevention.html. Accessed on Jan 31, 2019.

[9] The State of Obesity 2018: Better Policies for a Healthier America. https://www.tfah.org/report-details/the-state-of-obesity-20 18/ Accessed on Jan 16, 2019.

[10] Christakis NA, Fowler JH. The spread of obesity in a large social network over 32 years. N Engl J Med. 2007; 357: 370-379. Doi: 10.1056/NEJMsa066082.

[11] Ashlesha Datar, Nancy Nicosia. Assessing Social Contagion in Body Mass Index, Overweight, and Obesity Using a Natural Experiment. JAMA Pediatr. 2018; 172(3):239-246. Doi: 10.1001/jamapediatrics.2017.4882. Accessed June 8, 2019.

[12] American Medical Association House of Delegates. Recognition of obesity as a disease. Resolution 420 (A-13). http://www.npr.org/documents/2013/jun/ama-resolution-o besity.pdf. Accessed June 17, 2019.

[13] Bray GA, Kim KK, Wilding JPH. Obesity: a chronic relapsing progressive disease process. A position statement of the World Obesity Federation. Obes Rev. May 2017.

[14] Mechanick JI, Garber AJ, Handelsman Y, Garvey WT. American Association of Clinical Endocrinologists' position statement on obesity and obesity medicine. Endocr Pract.

[15] Guh DP, Zhang W, Bansback N, et al. The incidence of co-morbidities related to obesity and overweight: a systematic review and meta-analysis. BMC Public Health. 2009; 9: 88 .

[16] Puhl RM, Huer CA. Obesity stigma: important considerations for public health. Am J Pub Health. 2010; 100(6)1019-1028.

[17] What is a healthy eating style? ChooswMyPlat.gov. United States Department of Agriculture.https:/www.choosemyp late.gov/what-healthy-eating-style. Accessed on June 19, 2019 